\title{
Dynamic Resolution in GPU-Accelerated Volume Rendering to Autostereoscopic Multiview Lenticular Displays
}

\author{
Daniel Ruijters \\ X-Ray Predevelopment, Philips Healthcare, Veenpluis 4-6, 5680DA Best, The Netherlands \\ Correspondence should be addressed to Daniel Ruijters, danny.ruijters@philips.com \\ Received 12 December 2007; Revised 29 March 2008; Accepted 11 June 2008 \\ Recommended by Levent Onural
}

\begin{abstract}
The generation of multiview stereoscopic images of large volume rendered data demands an enormous amount of calculations. We propose a method for hardware accelerated volume rendering of medical data sets to multiview lenticular displays, offering interactive manipulation throughout. The method is based on buffering GPU-accelerated direct volume rendered visualizations of the individual views from their respective focal spot positions, and composing the output signal for the multiview lenticular screen in a second pass. This compositing phase is facilitated by the fact that the view assignment per subpixel is static, and therefore can be precomputed. We decoupled the resolution of the individual views from the resolution of the composited signal, and adjust the resolution on-the-fly, depending on the available processing resources, in order to maintain interactive refresh rates. The optimal resolution for the volume rendered views is determined by means of an analysis of the lattice of the output signal for the lenticular screen in the Fourier domain.
\end{abstract}

Copyright (C 2009 Daniel Ruijters. This is an open access article distributed under the Creative Commons Attribution License, which permits unrestricted use, distribution, and reproduction in any medium, provided the original work is properly cited.

\section{Introduction}

New developments in medical imaging modalities lead to ever increasing sizes in volumetric data. The ability to visualize and manipulate this $3 \mathrm{D}$ data interactively is of great importance in the analysis and interpretation of the data. Interactivity, in this context, means that the frame rates of the visualization are sufficient to provide direct feedback during user manipulation (such as rotating the scene). When the visualization's frame rate is too low, manipulation becomes very cumbersome. Five frames per second are often used as a required minimum frame rate in the medical world.

Direct volume rendering is a visualization technique that allows a natural representation, while maximally preserving the information encapsulated in the data. The interactive visualization of such data remains a challenge, since the frame rate is heavily depending on the amount of data to be visualized.

Autostereoscopic displays allow a stereoscopic view of a 3D scene, without the use of any additional aid, such as goggles. The additional depth impression that a stereoscopic image offers enables a natural interpretation of 3D data. Principally, there are two methods for conveying a stereo- scopic image: time multiplexing and spatial multiplexing of two or more views. Though two views are enough to create the impression of depth (after all, we have only two eyes), offering more views has the advantage that the viewer is not restricted to a fixed sweet spot, since there is a range of positions where the viewer will be presented with a stereoscopic visualization. As a consequence, multiple viewers can look at the same stereoscopic screen, without wearing goggles. Furthermore, it is possible to "look around" an object, when moving within the stereoscopic range, which aids the depth perception. The multiview lenticular display uses a sheet of lenses to spatially multiplex the views [1], and typically offer four to fifteen spatially sequential images.

The graphics processing unit (GPU) is a powerful parallel processor on today's off-the-shelf graphics cards. It is especially capable in performing single instruction multiple data (SIMD) operations on large amounts of data. In this article, a method for generating direct volume rendered images for display on lenticular screens is discussed, benefitting from the vast processing power of modern graphics hardware. The presented approach allows dynamical adjustment of the resolution of the volume rendered images, in order to guarantee a minimal frame rate. 


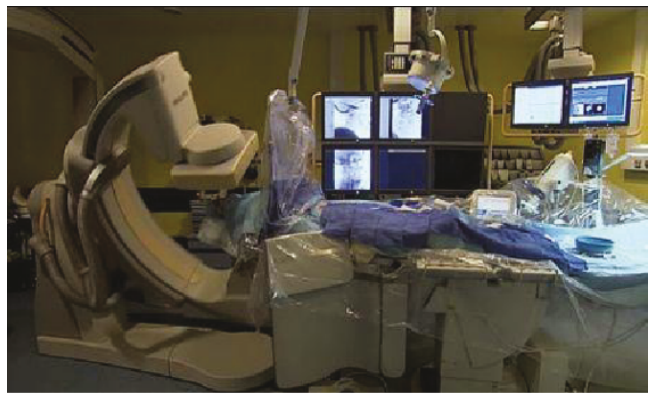

(a)

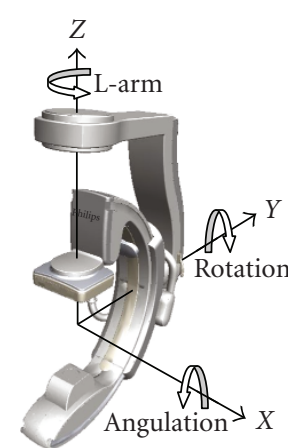

(b)

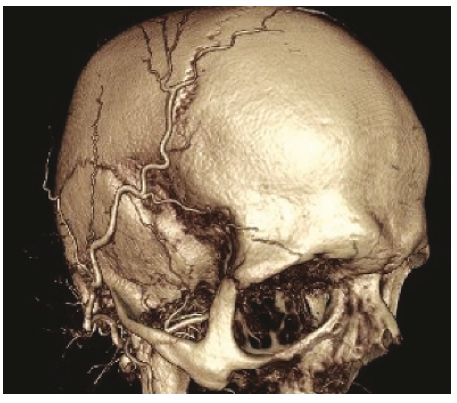

(c)

Figure 1: (a) The X-ray C-arm system in a clinical intervention. (b) The degrees of rotational freedom of the C-arm geometry. (c) A 3D data set, acquired using the X-ray C-arm system.

We applied the presented approach to visualize intraoperatively acquired 3D data sets on a Philips $42^{\prime \prime}$ lenticular screen, which was mounted in the operation room (OR). The orientation of the $3 \mathrm{D}$ data set followed in realtime the orientation of an X-ray C-arm system; see Figure 1. This approach aids in reducing the $\mathrm{X}$-ray radiation, since the physician can choose the optimal orientation to acquire Xray images without actually radiating. Further, it improves the interpretation of the live projective $2 \mathrm{D} \mathrm{X}$-ray image, which is presented on a separate display, since the 3D data set on the stereoscopic screen (which is in the same orientation) gives a proper depth impression through the stereovision of the lenticular screen. In this application, interactivity is very important, and the refresh rate has to be sufficient to provide a realtime impression. After all, when the refresh rate is too low, the interaction with the human operator can lead to oscillating manipulation. Further, the fact that the clinician is not limited to single sweet spot makes these displays particularly suitable for this environment, since the clinical intervention demands that the operator can be positioned freely in the range close to the patient.

\section{State of the Art}

In 1838, Sir Charles Wheatstone developed a device, called the stereoscope, which allowed the left and the right eyes to be presented with a different image (illustration or photograph), in order to create an impression of depth. Matusik and Pfister [2] presented a comprehensive overview of the various systems for stereoscopic visualizations that have been developed over the time. The development of auto stereoscopic display devices, presenting stereoscopic images without the use of glasses, goggles, or other viewing aids, has seen an increasing interest since the 1990s [3-5].

The advancement of large high resolution LCD grids, with sufficient brightness and contrast, has brought highquality multiview autostereoscopic lenticular displays within reach [6]. A number of publications have investigated the image quality aspects of autostereoscopic displays. Seuntiëns et al. [7] have discussed the perception quality of lenticular displays as a function of white noise. Konrad and Agniel
[8] describe the Fourier domain properties of the lenticular display, and they propose a pre-filtered sample approach. The effect of light that ought to be contributed to one particular view leaking into other views, which is called crosstalk, has been quantitatively investigated by Brasspenning et al. [9] and Boev et al. [10].

The range of viewing positions, allowing the perception of a stereoscopic image, is mainly determined by the number of views, offered by the display. Further, a higher resolution per view leads to less artifacts and improves the image quality. The required resolution of the LCD pixel grid can be established as the number of views times the resolution per view. Clearly, fulfilling both requirements demands very high resolution LCD pixel grids, which means that an enormous amount of pixel data has to be rendered and transferred to the display.

Several publications describe how the GPU can be employed to extract the data stream for the lenticular display from a 3D scene in an effective manner. Kooima et al. [11] present a two-pass GPU-based algorithm for two-view headtracked parallax barrier display. First the views for the left and the right eyes are rendered, and in the subsequent pass they are interweaved. Domonkos et al. [12] describe a twopass approach, dedicated for isosurface rendering. In the first pass they perform the geometry calculations on the pixelshader for every individual pixel, and in the second pass the shading is performed. Hübner and Pajarola [13] describe a GPU-based single-pass multiview volume rendering, varying the direction of the casted rays depending on their location on the lenticular screen.

The previous GPU-based approaches were dedicated render methods, working on the native resolution of the lenticular LCD grid. We present an approach that decouples the render resolution from the native LCD grid resolution, allowing lower resolutions, when higher frame rates are demanded.

\section{The Multiview Lenticular Display}

Multiview autostereoscopic displays can be regarded as three-dimensional light field displays [14, 15] (or fourdimensional, when also considering time). The dimensions 


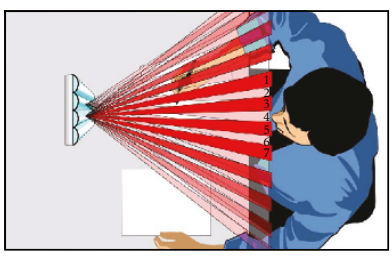

(a)

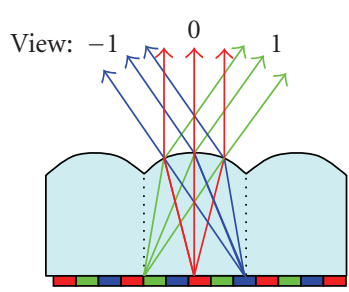

(b)
Figure 2: (a) The spatial multiplexing of the different views. (b) The light of the subpixels is directed into different directions by the lens [9].

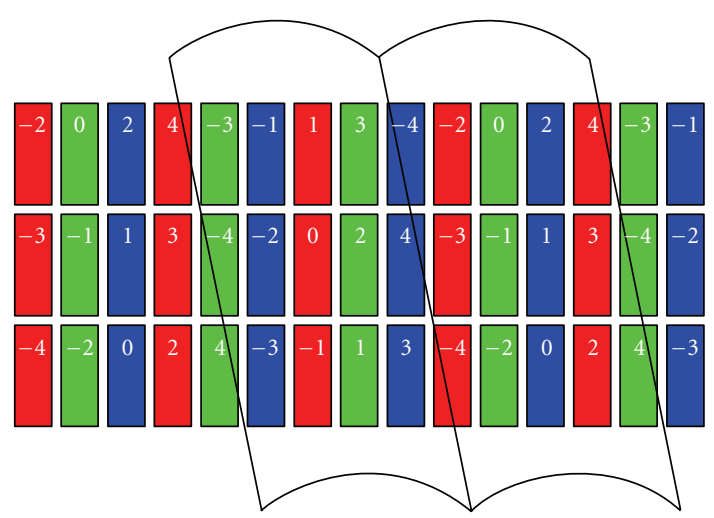

Figure 3: The cylindrical lenses depict every subpixel in a different view. The numbers in the subpixels indicate in which view they are visible.

are described by the parameters $(x, y, \phi)$, whereby $x$ and $y$ indicate a position on the screen and $\phi$ indicates the angle in the horizontal plane in which the light is emitted. The light is further characterized by its intensity and its color.

The multiview lenticular display device consists of a sheet of cylindrical lenses (lenticulars) placed on top of an LCD in such a way that the LCD image plane is located at the focal plane of the lenses [16]. The effect of this arrangement is that LCD pixels located at different positions underneath the lenticulars fill the lenses when viewed from different directions; see Figure 2. Provided that these pixels are loaded with suitable stereo information, a 3D stereo effect is obtained, in which the left and right eyes see different, but matching information. The screen we used offered nine distinct angular views, but our method is applicable to any number of views.

The fact that the different LCD pixels are assigned to different views (spatial multiplex) leads to a lower resolution per view than the resolution of the LCD grid [17]. In order to distribute this reduction of resolution over the horizontal and vertical axes, the lenticular cylindrical lenses are not placed vertically and parallel to the LCD column, but slanted at a small angle [1]. The resulting assignment of a set of LCD pixels, which is specified by the manufacturer, is illustrated in Figure 3. Note that the red, green, and blue color channels of a single pixel are depicted in different views.

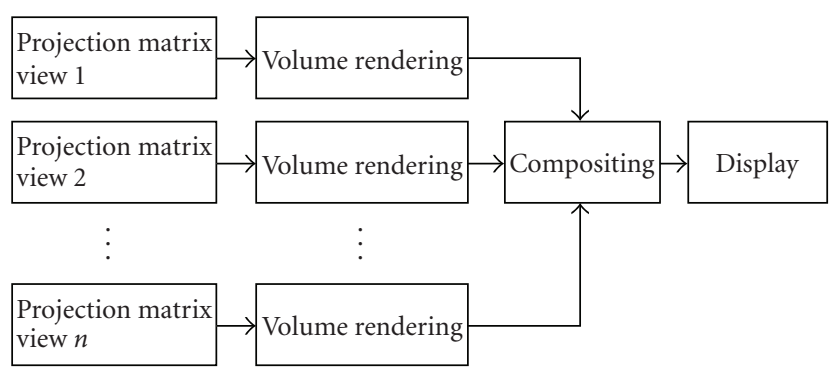

FIgURE 4: The process of rendering for the lenticular display. Optionally, the rendering of the $n$ individual views can be done in parallel.

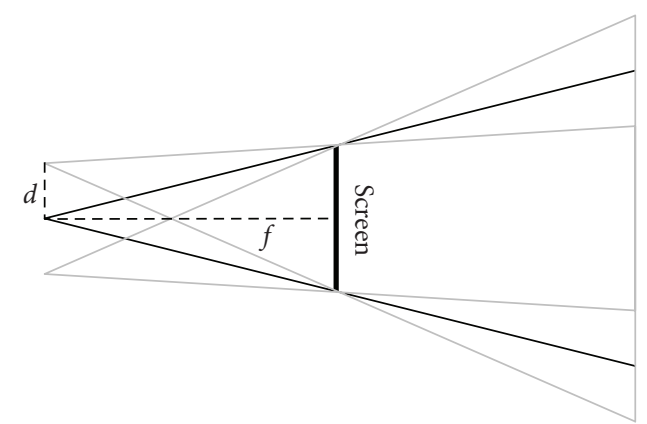

FIgURE 5: The frustums resulting from three different view points.

\section{The Different Angular Views}

We propose a two-pass algorithm: first the individual views from the different foci positions are separately rendered to an orthogonal grid. In the second pass, the final output signal has to be resampled from the views to a nonorthogonal grid in the compositing phase (see Figure 4). The processing power of the GPU is harvested for both passes. In order to maintain an acceptable frame rate, the resolution of the views can be changed dynamically.

The frustums that result from the different focal spots are illustrated in Figure 5. The viewing directions of the frustums are not parallel to the normal of the screen, except for the centered one. Therefore the corresponding frustums are asymmetric [18]. A world coordinate $(x, y, z)$ that is perspectively projected, using such an asymmetric frustum, leads to the following view port coordinate $v(x, y)$ :

$$
v(x, y)=\left(\frac{(x-n \cdot d) \cdot f}{f-z}+n \cdot d, \frac{y \cdot f}{f-z}\right),
$$

whereby $f$ denotes the focal distance, $n$ the view number, and $d$ the distance between the view cameras. All parameters should be expressed in the same metric (e.g., millimeters), and the origin is placed in the center of the view port.

Figures 5 and 6 illustrate the process of rendering the scene from focal spot positions with an offset to the center of the screen. After the projection matrix has been established, using (1), the scene has to be rendered for that particular view. All views are stored in a single texture, which we call texture1. In OpenGL, the views can be placed next to 


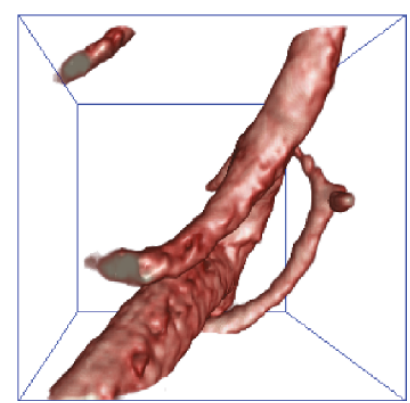

(a)

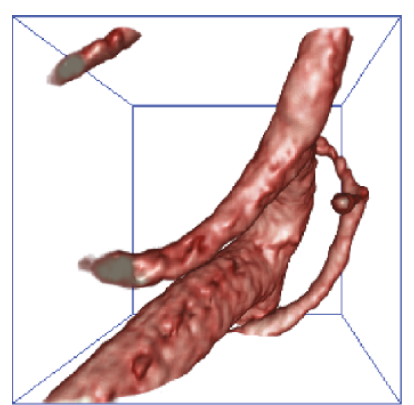

(b)
FIGURE 6: The same scene rendered from the most left and most right view points.

each other in horizontal direction, using the glViewport command. The location of a pixel in view $n$ in texture 1 can be found as follows:

$$
\vec{t}=\left(\frac{1}{2}+\frac{n}{\widetilde{N}}+\frac{2 p_{x}-1}{2 \tilde{N}}, p_{y}\right),
$$

whereby $\vec{t}$ denotes the normalized texture coordinate, $\vec{p}$ the normalized pixel coordinate within the view, and $\tilde{N}$ the total number of views. The view index $n$ is here assumed to be in the range $[-(\widetilde{N}-1) / 2,(\widetilde{N}-1) / 2]$, as is used in, for example, Figure 3.

\section{Direct Volume Rendering}

For each view the volumetric data set has to be rendered, using the appropriate frustum perspective projection. In order to use the GPU for volume rendering, the voxel data set has to be loaded in the texture memory of the graphics card. To obtain textures which are better suited for the memory architecture of the graphics hardware and to be able to deal with data set sizes exceeding the available texture memory, the data set is divided into the so-called bricks.

The actual direct volume rendering process consists of evaluating the volume rendering equation for rays which are casted through the pixels of display; see Figure 7. The volume rendering equation can be approximated by the following summation:

$$
i=\sum_{m=0}^{M}\left(\alpha_{m} c_{m} \cdot \prod_{m^{\prime}=0}^{m}\left(1-\alpha_{m^{\prime}}\right)\right)
$$

whereby $i$ denotes the resulting color of a ray, $\alpha_{m}$ the opacity at a given sample $m$, and $c_{m}$ the color at the respective sample. Since medical data sets typically only consist of scalar values, a transfer function has to be defined, mapping the scalar values to color and opacity values.

This summation can be broken down in $M$ iterations over the so-called over operator [19], whereby the rays are traversed in a back-to-front order:

$$
C_{m+1}=\alpha_{m} \cdot c_{m}+\left(1-\alpha_{m}\right) \cdot C_{m}
$$

Here $C_{m}$ denotes the intermediate value for a ray. For (4), standard alpha blending, offered by DirectX or OpenGL, can

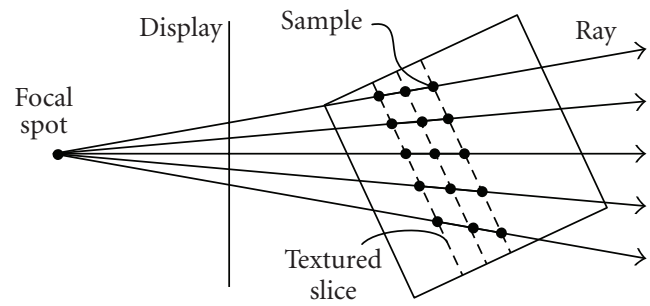

(a)

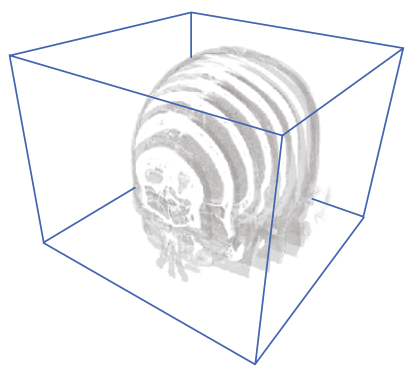

(b)

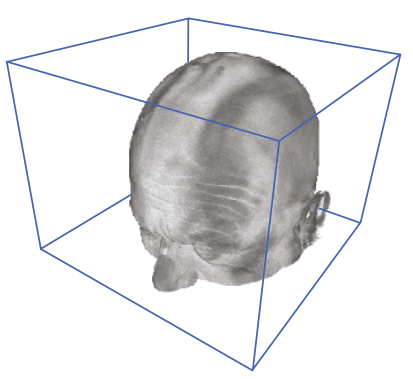

(c)
Figure 7: (a) Volume rendering involves the evaluation of the volume render equation along the rays, passing through the pixels of the display. The usage of textured slices means that the rays are not evaluated sequentially. Rather for a single slice the contribution of the sample points to all rays is processed. (b) A volume rendered data set, with large intervals between the textured slices. (c) The same volume rendered data set, with a small distance between the textured slices.

be used. In order to execute (4), a set of textured slices, containing the volumetric medical data, are blended into each other [20]. To map the slices properly on the display area, the projection matrix is set to match the perspective defined by the focal spot and the display area; see (1). The modelview matrix is a rigid transformation matrix, and determines the position, orientation, and scale of the volume in the 3D space. The slices are then processed in a back-tofront order, whereby the intermediate results $C_{m}$ are written in the frame buffer.

Our GPU-based direct volume rendering implementation is capable of rendering highlights, diffuse, and ambient lighting, which enhances the depth impression. Further it can handle any perspective projection matrix.

The rendered views are stored locally on the graphics card. They are put horizontally next to each other, in a single wide rectangular texture map; see Section 4 . The fact that the entire scene has to be drawn multiple times is partially compensated by the fact that the individual views have a lower resolution than the output window.

\section{Resolution Considerations}

The maximum information density that can be conveyed by the lenticular display per view is determined by the way the pixels of the LCD grid are refracted by the lenticular lenses. In modern lenticular displays, the lens array is slanted under a slight angle, which affects the distribution of the set of pixels that are diverted to a particular viewing angle. 
In Figure 9(a), it is shown how the green subpixels, visible from the middle viewing position (view 0), are distributed over the LCD grid. Though the allocation of the subpixels over the grid is regular, it is not orthogonal. The sampling theory of multidimensional signals, described by Dubois [21], can be used to examine the frequency range that can be transmitted by a certain nonorthogonal grid. Especially the maximum view port size that does not lead to aliasing is of interest. When the resolution of the view port is too high, the compositing undersamples the view, and aliasing occurs. Though such views can be low-pass filtered to prevent aliasing, it is preferable to render them immediately at the optimal resolution, in order to keep the load on the scarce processing resources as low as possible.

The set of subpixels that are refracted to the same angular view can be considered to form a lattice. Let the vectors $\left\{\vec{v}_{1}, \vec{v}_{2}, \ldots, \vec{v}_{N}\right\}$ form a basis, not necessarily orthogonal, of $\mathbb{R}^{N}$. Then lattice $\Lambda \subset \mathbb{R}^{N}$ is defined as a set of discrete points in $\mathbb{R}^{N}$, formed by all linear combinations of vectors $\vec{v}_{1}, \vec{v}_{2}, \ldots, \vec{v}_{N}$ with integer coefficients.

In order to perform a Fourier transform of a signal, sampled on a lattice, the reciprocal lattice is required. The reciprocal lattice $\Lambda^{*}$ of lattice $\Lambda$ is defined as the set of vectors $\vec{y}$, such that $\vec{y} \cdot \vec{x}$ is an integer for all $\vec{x} \in \Lambda$. Let $V$ be the matrix, whose columns are the representation of the basis vectors $\vec{v}_{1}, \vec{v}_{2}, \ldots, \vec{v}_{N}$ in the standard orthonormal basis for $\mathbb{R}^{N}$. Then matrix $W$, containing the basis vectors of the reciprocal lattice $\Lambda^{*}$, is determined by $W^{T} V=I$, with $I$ being the $N \cdot N$ identity matrix.

The Voronoi cell of a lattice is defined as the set of all points in $\mathbb{R}^{N}$ closer to origin $\overrightarrow{0}$ than to any other lattice point; see Figure 8 . The basis $V$ for a given lattice is not unique (i.e., a lattice $\Lambda$ can be described by several different basis matrices $V)$. However, any basis for a certain lattice $\Lambda$ delivers the same unique Voronoi cell.

Let the Fourier transform of a continuous multidimensional signal $u_{c}(\vec{x})$ with $\vec{x} \in \mathbb{R}^{N}$ be defined as

$$
U_{c}(\vec{f})=\int_{\mathbb{R}^{N}} u_{c}(\vec{x}) e^{-j 2 \pi \vec{f} \cdot \vec{x}} d \vec{x}, \quad \vec{f} \in \mathbb{R}^{N} .
$$

The Fourier transformation of signal $u_{c}$ sampled on lattice $\Lambda$ is periodical, with lattice $\Lambda^{*}$ as periodicity [21]:

$$
U(\vec{f})=\frac{1}{|\operatorname{det} V|} \sum_{\vec{r} \in \Lambda^{*}} U_{c}(\vec{f}+\vec{r}) .
$$

Consequently, if a signal that is not bandwidth limited within the Voronoi cell of lattice $\Lambda^{*}$ is sampled on lattice $\Lambda$, spectral overlap (i.e., aliasing) occurs.

The sampling that occurs in the compositing phase can be examined, considering only one monochromatic primary color (red, green, or blue), or can be evaluated for all colors together; see Figure 9. The basis matrices $V$ of the sample lattice can be established by taking two vectors (nonlinearly dependent) between adjacent lattice points. The LCD pixel distance is used as a metric, which means that two neighboring subpixels (e.g., red and green) have a distance of $1 / 3$ pixel. For example, for the color-independent lattice

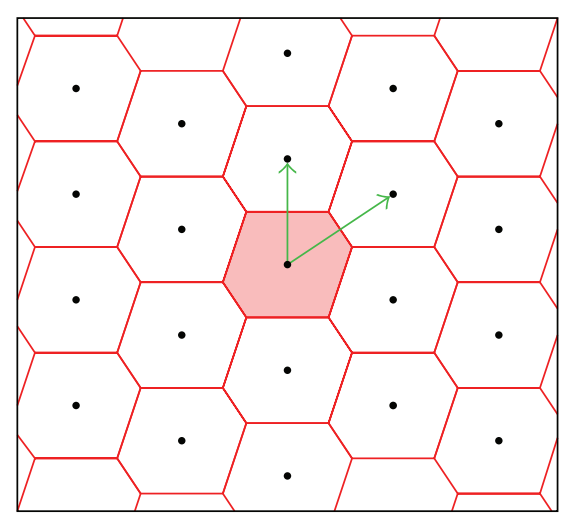

Figure 8: A lattice (black dots) and corresponding Voronoi cells (red). The green vectors compose a possible basis $V$ for this lattice. The Voronoi cell of a given lattice point is the set of points in $\mathbb{R}^{N}$ that are closer to this particular lattice point than to any other lattice point.

(Figure $9(\mathrm{~b})$ ), we take the vectors $\vec{v}_{1}=(5 / 3,-1)^{T}$ and $\vec{v}_{2}=$ $(4 / 3,1)^{T}$. This delivers the following basis matrices $V$ and their reciprocals $W^{T}$ :

$$
\begin{array}{cc}
V_{\text {mono }}=\left(\begin{array}{cc}
3 & -1 \\
0 & -3
\end{array}\right), & V_{\text {color }}=\left(\begin{array}{cc}
\frac{5}{3} & \frac{4}{3} \\
-1 & 1
\end{array}\right), \\
W_{\text {mono }}=\frac{1}{9}\left(\begin{array}{cc}
3 & 0 \\
-1 & -3
\end{array}\right), & W_{\text {color }}=\frac{1}{9}\left(\begin{array}{cc}
3 & 3 \\
-4 & 5
\end{array}\right) .
\end{array}
$$

The individual views are rendered on an orthogonal grid, and the Voronoi cell of an orthogonal lattice is a simple rectangle. The maximum resolution that can be visualized on the lenticular screen can be examined by fitting this Nyquist frequency rectangle range of the orthogonal grid on the Voronoi cell of the reciprocal lattice of the lenticular sample grid.

A logical choice for the resolution of the individual views, for a lenticular screen with 9 views, seems to be $1 / 3$ of the LCD pixel grid resolution in both directions. After all, this represents the same amount of information: 9 views with each $1 / 3 \cdot 1 / 3$. the amount of pixels of the LCD grid. We call this the $1 / 3$ orthogonal grid. The Nyquist frequency rectangle of this resolution has been depicted on top of the Voronoi cell of the reciprocal lattice of the lenticular sample grid in Figure 9. Looking at a single primary color channel (in Figure 9(a) the green subpixels are used, but the lattice is the same for red and blue), it can be noted that the rectangle is not completely encapsulated within the Voronoi cell. This means that for monochromatic red, green, and blue images there is a slight undersampling in certain directions, and aliasing might occur in the higher frequencies. If the lenticular lattice for a single view is considered, regardless of the colors of the subpixels, then the rectangle is completely contained within the Voronoi cell; see Figures 9(b) and 9(d). This implies that for gray colored images there is no aliasing when only the intensities are considered, but there might be some aliasing between the colors. In practise, this behavior resembles color dithering for real-world images. 


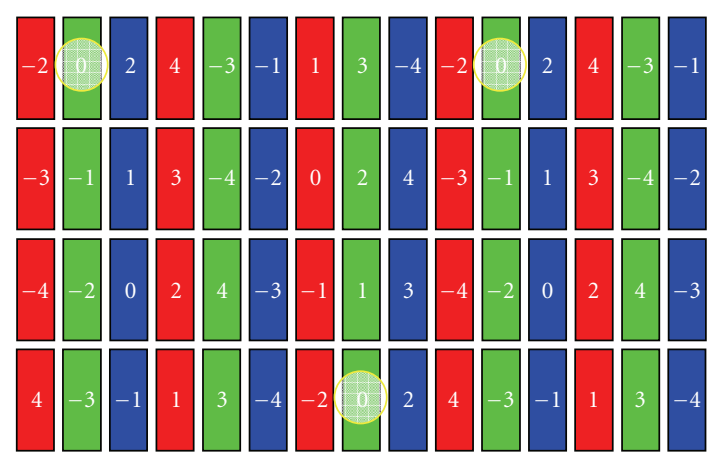

(a)

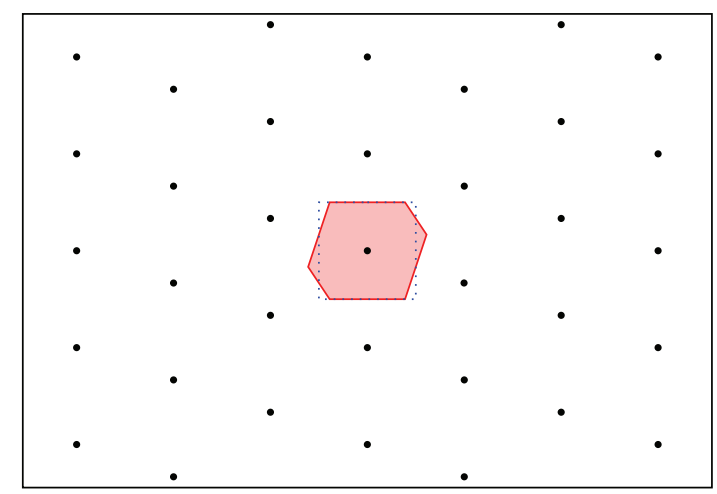

(c)

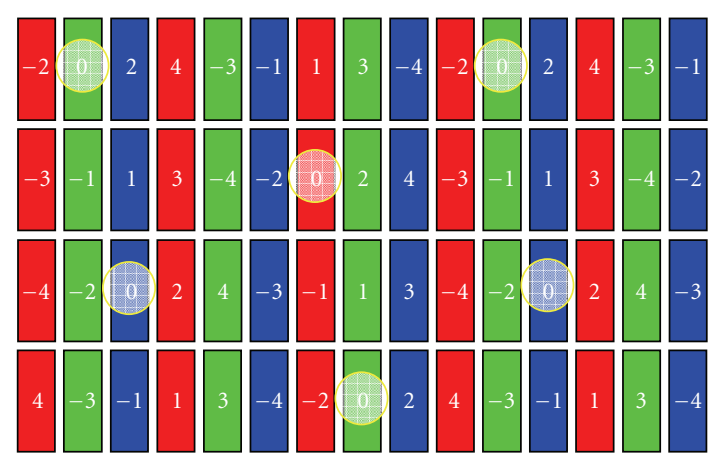

(b)

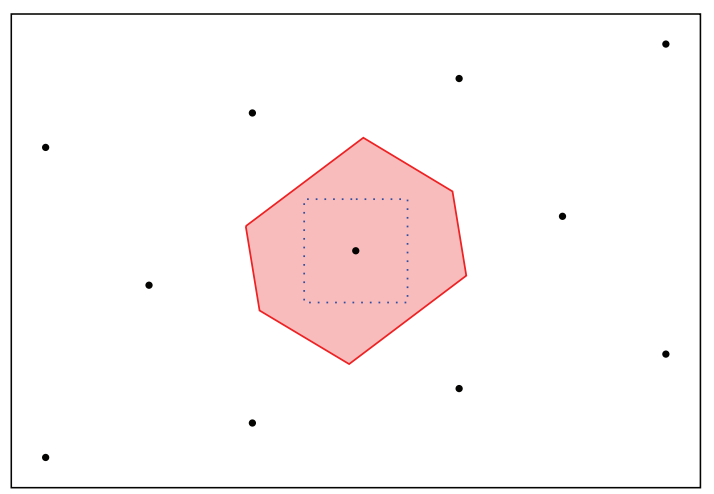

(d)

FIgURE 9: (a) The LCD pixel grid and the view that is associated with each subpixel. The green subpixels that are diverted to view 0 are circled. (b) All subpixels that are diverted to view 0 are circled, independent from their color. (c) The reciprocal lattice of the green subpixels for view 0 . The Voronoi cell of the reciprocal lattice is indicated in pink. In blue, the Nyquist frequency of the $1 / 3$ orthogonal grid is indicated. Since the Voronoi cell does not cover the complete Nyquist frequency range, slight aliasing in the higher frequencies might occur. (d) The reciprocal lattice of the subpixel configuration of view 0 , ignoring their color. Since the Nyquist frequency range (blue) is contained within the Voronoi cell (pink), there is no aliasing in the intensity image.

High frequent primary-colored structures (such as thin lines) may suffer from slight visible aliasing artifacts, though.

\section{Dynamic Resolution}

As long as there are sufficient processing resources available, the resolution of the angular views is set to the $1 / 3$ orthogonal grid. This resolution provides a good tradeoff between maximum detail and minimum aliasing, as described above. When the frame rate falls below a predefined threshold, the resolution of the individual views can be lowered; see Figure 10.

For the sake of simplicity, we use the same resolution for all views that contribute to a particular frame, but there is no technical reason imposing this. The resolution of a view can simply be changed by setting the view port to the desired size. The size of the offscreen buffer containing texturel is not changed; it is always kept at the maximum size needed.

Of course, lowering the view resolution does not guarantee that the desired minimum frame rate is achieved. This is mostly determined by the major bottlenecks in the $3 \mathrm{D}$ scene [20]. In cases where the major bottleneck is determined by the fragment throughput, the frame rate scales very well with the view port size, and may increase significantly. When, for example, the vertex throughput is the most important bottleneck, the frame rate is largely independent of the view port size.

Lower-resolution views correspond to smaller Nyquist rectangles in the frequency domain. For lower resolutions, the rectangle typically fits in the Voronoi cell of Figure 9(c), which implies that the view is oversampled by the compositing process. This corresponds to low-pass filtering the view at maximum resolution, which means that reducing dynamically the view resolution does not lead to aliasing artifacts, but merely to loss of detail. These details can be regained when the scene content is more static, and there is sufficient time to render the scene at high resolution.

To composite the final image, which will be displayed on the lenticular screen, the red, green, and blue components of each pixel have to be sampled from a different view (see Figure 3). The view number stays fixed all the time for each subpixel. Therefore, this information is precalculated once, and then put in a static texture map, called textureo.

In the compositing phase, all the pixels in the output image are parsed by a GPU program. For each normalized pixel coordinate $\vec{p}$ in the output image, texture 0 will deliver 


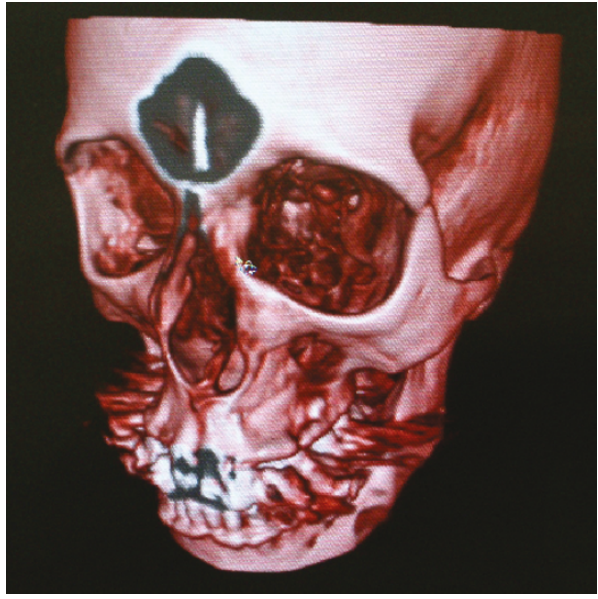

(a)

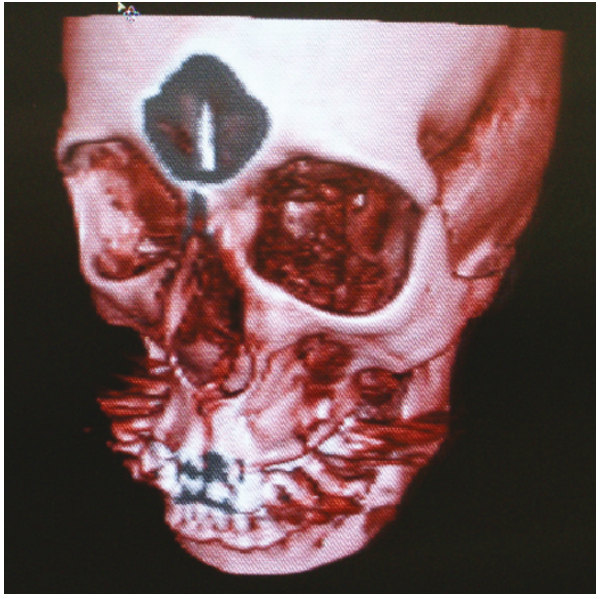

(b)

Figure 10: (a) The lenticular screen has been photographed to show how a view is being displayed, rendered at the $1 / 3$ orthogonal grid resolution. (b) The same view, but sampled at 0.375 the resolution of the view in (a). Though the downsampling is visible, the effect is less strong than might be expected. This can be contributed to the fact that the displaying process possesses a low-pass filter character, due to effects like crosstalk.

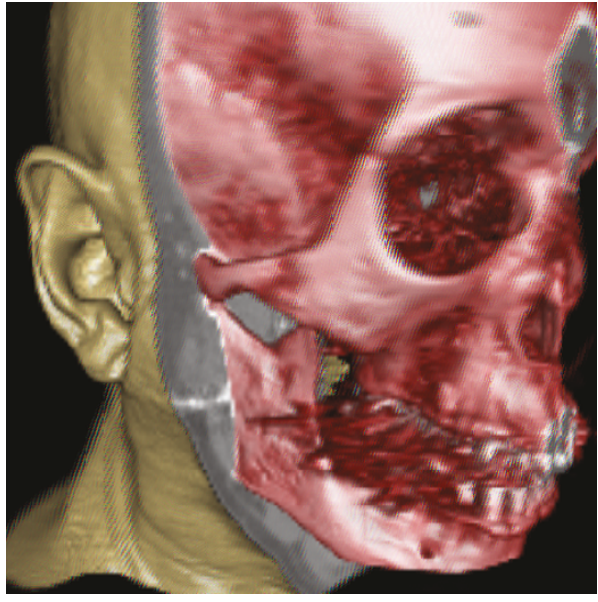

(a)

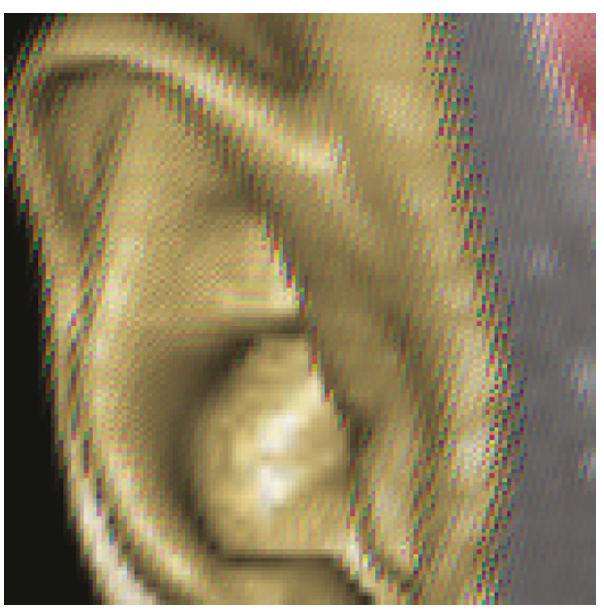

(b)

Figure 11: (a) The raw output signal that is sent to the lenticular display. Please note that the Moiré-like structures are not artifacts, but can be contributed to the interweaved subpixels, belonging to different views. (b) A zoomed fragment of the left image.

the view numbers $n$ that have to be sampled for the red, green, and blue components. The respective views are then sampled in texture 1 according to (2), using bilinear interpolation, delivering the appropriate pixel value; see Figure 11.

\section{Results and Conclusions}

Figure 12 shows the adaptive adjustment of the view resolution. The minimum desired frame rate was set to 7 frames per second in this case, which corresponds to rendering 63 views per second, since the lenticular display requires nine views to compose one frame. The measurements were performed using volume rendering of the data set depicted in Figure 10, and involved advanced lighting. It consisted of
$256^{2} \cdot 200$ voxels $(25 \mathrm{MB})$, while the output signal comprised $1600 \cdot 1200$ pixels. The resolution of the views was the resolution of the $1 / 3$ orthogonal grid, multiplied by the scaling factor (right vertical axis) in both the $x$ - and $y$ directions.

In order to characterize the performance of the GPUaccelerated volume rendering and compositing, several data sets were rendered at the $1 / 3$ orthogonal grid resolution to an output window of $800^{2}$ pixels. Nine views were rendered per frame, and the view size was $264^{2}$ pixels. Using a scan of a foot, consisting of $256^{2} \cdot 200$ voxels $(25 \mathrm{MB})$, we achieved a frame rate of 52.4 frames per second, which corresponds to rendering 471 views per second. A CT scan of a head of $512^{2} .256$ voxels $(128 \mathrm{MB})$ could be rendered at 19.2 frames per second (173 views per second), and a 3DRA data set of 


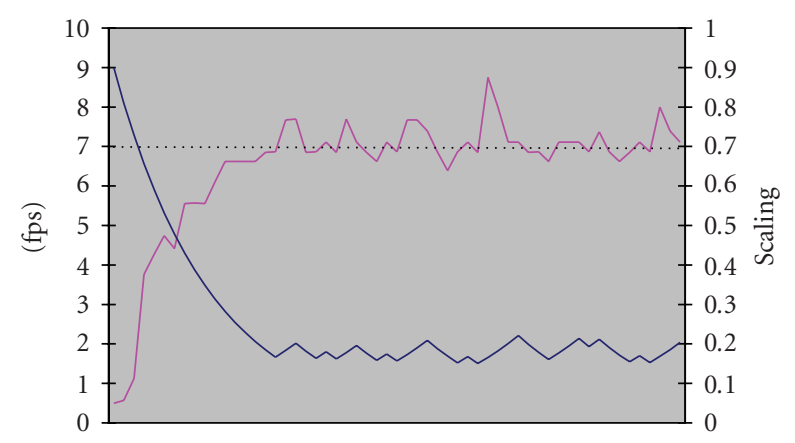

Figure 12: Pink line: frame rate in frames per second (fps). Blue line: view resolution scaling. The horizontal axis represents the time.

$512^{3}$ voxels $(256 \mathrm{MB})$, showing the vasculature in the brain, could be visualized at 21.3 frames per second (192 views per second).

All measurements were obtained, using a $2.33 \mathrm{GHz}$ Pentium 4 system, with 2 GB RAM memory, and an nVidia QuadroFX 3500 with $256 \mathrm{MB}$ on board memory as graphics card.

In this article, a method for accelerated direct volume rendering to multiview lenticular displays has been presented. Due to the GPU-acceleration, together with the adaptive adjustment of the intermediate view resolution, interactive frame rates can be reached, which allows intuitive manipulation of the rendered scene. Since both the volume rendering and the compositing take place on the graphics hardware, the requirements for the other components of the PC system are rather modest. Thus the realization of the proposed high-performance system can be very cost effective.

The fact that viewers do not need to wear any additional glasses, and are not limited to a sweet spot, as well as the fact that large data sets can be manipulated interactively, make this method very suitable for a clinical interventional environment.

\section{References}

[1] C. van Berkel, D. W. Parker, and A. R. Franklin, "Multiview 3D LCD," in Stereoscopic Displays and Virtual Reality Systems III, vol. 2653 of Proceedings of SPIE, pp. 32-39, San Jose, Calif, USA, January-February 1996.

[2] W. Matusik and H. Pfister, "3D TV: a scalable system for realtime acquisition, transmission, and autostereoscopic display of dynamic scenes," ACM Transactions on Graphics, vol. 23, no. 3, pp. 814-824, 2004.

[3] M. Halle, "Autostereoscopic displays and computer graphics," ACM Computer Graphics, vol. 31, no. 2, pp. 58-62, 1997.

[4] N. A. Dodgson, "Autostereoscopic 3D displays," Computer, vol. 38, no. 8, pp. 31-36, 2005.

[5] L. Onural, T. Sikora, J. Ostermann, A. Smolic, M. R. Civanlar, and J. Watson, "An assessment of 3DTV technologies," in Proceedings of the 60th Annual NAB Broadcast Engineering Conference, pp. 456-467, Las Vegas, Nev, USA, April 2006.

[6] A. Vetro, W. Matusik, H. Pfister, and J. Xin, "Coding approaches for end-to-end 3D TV systems," in Proceedings of the 24th Picture Coding Symposium (PCS '04), pp. 319-324, San Francisco, Calif, USA, December 2004.

[7] P. J. H. Seuntiëns, I. E. J. Heynderickx, W. A. IJsselsteijn, et al., "Viewing experience and naturalness of 3D images," in Three-Dimensional TV, Video, and Display IV, vol. 6016 of Proceedings of SPIE, pp. 43-49, Boston, Mass, USA, October 2005.

[8] J. Konrad and P. Agniel, "Subsampling models and anti-alias filters for 3-D automultiscopic displays," IEEE Transactions on Image Processing, vol. 15, no. 1, pp. 128-140, 2006.

[9] R. Braspenning, E. Brouwer, and G. de Haan, "Visual quality assessment of lenticular based 3D displays," in Proceedings of the 13th European Signal Processing Conference (EUSIPCO '05), Antalya, Turkey, September 2005.

[10] A. Boev, A. Gotchev, and K. Egiazarian, "Crosstalk measurement methodology for autostereoscopic screens," in Proceedings of the 3DTV Conference, pp. 1-4, Kos Island, Greece, May 2007.

[11] R. L. Kooima, T. Peterka, J. I. Girado, J. Ge, D. J. Sandin, and T. A. Defanti, "A GPU sub-pixel algorithm for autostereoscopic virtual reality," in Proceedings of the IEEE Virtual Reality (VR '07), pp. 131-137, Charlotte, NC, USA, March 2007.

[12] B. Domonkos, A. Egri, T. Fóris, T. Juhász, and L. SzirmayKalos, "Isosurface ray-casting for autostereoscopic displays," in Proceedings WSCG, pp. 31-38, Plzen, Czech Republic, January-February 2007.

[13] T. Hübner and R. Pajarola, "Single-pass multiview volume rendering," in Proceedings of the IADIS International Conference on Computer Graphics and Visualization (CGV '07), Lisbon, Portugal, July 2007.

[14] M. Levoy and P. Hanrahan, "Light field rendering," in Proceedings of the 23rd Annual Conference on Computer Graphics and Interactive Techniques (SIGGRAPH '96), pp. 31-42, New Orleans, La, USA, August 1996.

[15] A. Isaksen, L. McMillan, and S. J. Gortler, "Dynamically reparameterized light fields," in Proceedings of the 27th Annual Conference on Computer Graphics and Interactive Techniques (SIGGRAPH '00), pp. 297-306, New Orleans, La, USA, July 2000.

[16] C. van Berkel, "Image preparation for 3D LCD," in Stereoscopic Displays and Virtual Reality Systems VI, vol. 3639 of Proceedings of SPIE, pp. 84-91, San Jose, Calif, USA, January 1999.

[17] N. A. Dodgson, "Autostereo displays: 3D without glasses," in Proceedings of the Electronic Information Displays (EID '97), Surrey, UK, November 1997.

[18] D. Maupu, M. H. Van Horn, S. Weeks, and E. Bullitt, "3D stereo interactive medical visualization," IEEE Computer Graphics and Applications, vol. 25, no. 5, pp. 67-71, 2005.

[19] T. Porter and T. Duff, "Compositing digital images," ACM Computer Graphics, vol. 18, no. 3, pp. 253-259, 1984.

[20] D. Ruijters and A. Vilanova, "Optimizing GPU volume rendering," Journal of WSCG, vol. 14, no. 1-3, pp. 9-16, 2006.

[21] E. Dubois, "The sampling and reconstruction of time-varying imagery with application in video systems," Proceedings of the IEEE, vol. 73, no. 4, pp. 502-522, 1985. 